\title{
Equilibrative Nucleoside Transporter 2 Regulates Associative Learning and Synaptic Function in Drosophila
}

\author{
David Knight, ${ }^{1}$ Philip J. Harvey, ${ }^{1 \star}$ Konstantin G. Iliadi, ${ }^{1 \star}$ Markus K. Klose, ${ }^{1}$ Natalia Iliadi, ${ }^{1}$ Eva Dolezelova, ${ }^{2}$ \\ Milton P. Charlton, ${ }^{3}$ Michal Zurovec, ${ }^{2}$ and Gabrielle L. Boulianne ${ }^{1,4}$ \\ ${ }^{1}$ Program in Developmental and Stem Cell Biology, The Hospital for Sick Children, Toronto, Ontario M5G 1L7, Canada, ${ }^{2}$ Institute of Entomology and \\ University of South Bohemia, CZ-37005 Ceske Budejovice, Czech Republic, and Departments of ${ }^{3}$ Physiology and ${ }^{4}$ Molecular Genetics, University of \\ Toronto, Toronto, Ontario M5S 1A8, Canada
}

\begin{abstract}
Nucleoside transporters are evolutionarily conserved proteins that are essential for normal cellular function. In the present study, we examined the role of equilibrative nucleoside transporter 2 (ent2) in Drosophila. Null mutants of ent 2 are lethal during late larval/early pupal stages, indicating that ent 2 is essential for normal development. Hypomorphic mutant alleles of ent2, however, are viable and exhibit reduced associative learning. We additionally used RNA interference to knock down ent 2 expression in specific regions of the CNS and show that ent 2 is required in the $\alpha / \beta$ lobes of the mushroom bodies and the antennal lobes. To determine whether the observed behavioral defects are attributable to defects in synaptic transmission, we examined transmitter release at the larval neuromuscular junction (NMJ). Excitatory junction potentials were significantly elevated in ent2 mutants, whereas paired-pulse plasticity was reduced. We also observed an increase in stimulus dependent calcium influx in the presynaptic terminal. The defects observed in calcium influx and transmitter release probability at the NMJ were rescued by introducing an adenosine receptor mutant allele (AdoR $\left.{ }^{1}\right)$ into the ent2 mutant background. The results of the present study provide the first evidence of a role for ent 2 function in Drosophila and suggest that the observed defects in associative learning and synaptic function may be attributable to changes in adenosine receptor activation.
\end{abstract}

\section{Introduction}

By acting as energy transfer molecules (e.g., ATP and GTP) and precursors for nucleic acid synthesis, nucleosides play an important role in many cellular homeostatic processes (Rose and Coe, 2008). Additionally, adenosine can act on $P_{1}$ purinergic receptors to modulate neuronal and endocrine signaling (Burnstock, 2008). Nucleoside transport across cell membranes is mediated by two evolutionarily distinct gene families. The equilibrative nucleoside transporters (ENTs) facilitate passive bidirectional nucleoside transport, whereas the concentrative nucleoside transporters (CNTs) facilitate unidirectional $\mathrm{Na}^{+}$-dependent nucleoside transport (Pastor-Anglada et al., 2008; Young et al., 2008). In mammals, four ENTs have been identified. ENT1, ENT2, and ENT4 are reported to show plasma membrane localization, whereas ENT3 appears to be localized to intracellular structures (Young et al., 2008).

As indicated above, both ENTs and CNTs play a key role in the regulation of purinergic (adenosine and ATP) receptor signaling.

Received Dec. 16, 2009; revised Feb. 4, 2010; accepted March 1, 2010.

This work was supported by Canadian Institutes of Health Research Grants MOP 14143 and MOP 67130 (G.L.B.) and by Research Center Program of Ministry of Education, Youth, and Sports Grant LC06077 (M.Z.). D.K., P.J.H., and M.K.K. are recipients of Restracomp Fellowship Awards from the Hospital for Sick Children. G.L.B. is the recipient of a Tier 1 Canada Research Chair in Molecular and Developmental Neurobiology. We thank Dr. W. S. Trimble, Dr. T. DaSylva, and B. Chow for helpful discussions and critical comments on this manuscript. We also thank E.M.C. Skoulakis and G. Roman for the $0 K 66^{\text {Gal4 }}$ and $17 D^{\text {Gal4 }}$ lines, respectively.

*P.J.H. and K.G.I. contributed equally to this work.

Correspondence should be addressed to Gabrielle L. Boulianne at the above address. E-mail: gboul@sickkids.ca. DOI:10.1523/JNEUROSCI.6241-09.2010

Copyright $\odot 2010$ the authors $\quad 0270-6474 / 10 / 305047-11 \$ 15.00 / 0$
In the CNS, ENT1 expression can modulate behaviors such as anxiety and drug dependence/addiction by regulating the availability of adenosine for $\mathrm{P}_{1}$ purinergic receptors in different regions of the CNS (Alanko et al., 2003; Choi et al., 2004; Guillén-Gómez et al., 2004). ENTs and CNTs have also been shown to act as routes of entry for drugs used to treat cancer and parasitic infections like malaria (Zhang et al., 2007; Downie et al., 2008). Given this therapeutic potential for ENTs and CNTs, a more complete understanding of the multiple roles played by these proteins will be important.

Model organisms such as Drosophila melanogaster have proven to be valuable research tools because of greater genetic tractability and less genetic redundancy than mammalian models. Three ENTs have been identified in Drosophila (Sankar et al., 2002); however, when expressed in Xenopus oocytes, only ent2 showed nucleoside transport activity (Machado et al., 2007). Two CNTs have also been identified in Drosophila (Machado et al., 2007), although the functional properties of these transporters remains uncertain. Another appealing feature is the fact that Drosophila possesses a single $\mathrm{P}_{1}$ (adenosine) receptor gene (AdoR) (Dolezelova et al., 2007) and no $\mathrm{P}_{2}$ (ATP) receptors (Littleton and Ganetzky, 2000; Lima and Miesenböck, 2005). Together, these results suggest that Drosophila could be a useful model organism for studying both the role of equilibrative nucleoside transporters in different physiological processes and the interaction between equilibrative nucleoside transport and adenosine receptor signaling.

In the present study, we show that null mutants for ent 2 are lethal indicating an essential role for ent2 during Drosophila de- 
velopment. We also show that hypomorphic mutations in ent2 give rise to defects in associative learning in Drosophila. Moreover, our data show that ent2 is required in the $\alpha / \beta$ lobes of the mushroom bodies and antennal lobes for normal associative learning. We additionally show that ent 2 regulates presynaptic calcium influx leading to defects in synaptic strength and plasticity at the neuromuscular junction. Finally, we provide evidence of a genetic interaction between ent 2 and AdoR and show that the changes in synaptic function observed in ent 2 mutants may be mediated by changes in adenosine receptor activation.

\section{Materials and Methods}

Fly stocks. The P124 insertion mutant (referred to as ent ${ }^{\mathrm{P} 124}$ ) was generated in a screen looking for genes involved in learning or memory (Kamyshev et al., 2000; Bragina and Kamyshev, 2003). The ent2 ${ }^{\mathrm{P} 124}$ insertion site was shown by sequencing to be $21 \mathrm{bp}$ downstream of the predicted transcription start site of ent2. The ent $2^{1}$, ent $2^{2}$, and ent $2^{3}$ mutant alleles were generated by imprecise excision of the ent $2^{\mathrm{P} 124}$ transposable element. The ent $2^{1}$ excision removes the entire coding region of ent 2 as well as most of the $5^{\prime}$ - and $3^{\prime}$ - untranslated regions (UTRs), leaving $\sim 10 \mathrm{bp}$ of the $5^{\prime}$-UTR and $60 \mathrm{bp}$ of the $3^{\prime}$-UTR. As indicated in Figure $1 \mathrm{~A}, \mathrm{CG} 13766$ overlaps the $3^{\prime}$ end of ent 2 by $\sim 30 \mathrm{bp}$. The ent $2^{1}$ excision does not remove any of the CG13766 gene. The ent $2^{2}$ excision removes all but the first $21 \mathrm{bp}$ of the $5^{\prime}$-UTR and the first $73 \mathrm{bp}$ of the coding sequence. The ent $2^{3}$ excision removes the first 20 bp of the $5^{\prime}$ UTR of ent 2 as well as $227 \mathrm{bp}$ of the intergenic region between ent 2 and CG9596. The adenosine receptor mutant $\left(A d o R^{1}\right)$ was generated by homologous recombination and includes a stop codon after amino acid 278 (Dolezal et al., 2005). All stocks were outcrossed for at least five generations to the cantonized $w^{1118}$ strain before experimentation to reduce genetic variability. The UAS-ent2-RNAi line (termed ent $2^{\mathrm{RNAi}}$ ) was obtained from the Vienna RNAi Stock Center (stock number 7618). The NP7068 line (termed ent $2^{\text {Gal4 }}$ ) contains a $\mathrm{P}\{\mathrm{GawB}\}$ insertion $7 \mathrm{bp} 5^{\prime}$ of the ent $2^{\mathrm{P} 124}$ insertion and was obtained from the Kyoto Stock Center. The OK66 ${ }^{\text {Gal4 } 4}$ line was a gift from E. M. C. Skoulakis, (Texas A\&M University, College Station, TX), and the $17 \mathrm{D}^{\text {Gal4 }}$ line was a gift from G. Roman (University of Houston, Houston, TX). The $739^{\text {Gal4 }}$ line and two deficiency lines [Df(2L)BSC6 and Df(2L)BSC7] that cover the $26 \mathrm{E} 3$ region of the second chromosome were obtained from the Bloomington Stock Center. The UAS- $d n c$ line has been previously described (Cheung et al., 1999).

Quantitative reverse transcription- $P C R$. Total RNA was extracted from adult flies using Trizol reagent and purified using Mini RNA isolation columns from Invitrogen. Isolated RNAs were DNase treated using Invitrogen amplification grade DNase I. DNase-treated RNA was reverse transcribed using a reverse transcription kit from Roche with $250 \mathrm{ng}$ random hexamers. The cDNA was amplified by quantitative PCR using the Roche Light Cycler 480 and the following primers: ent2 Fwd, CGCGCAGGGAAAATCAAACC; ent2 Rev, GCCAACACCCACGTTGATCC; CG9596 Fwd, AACCTGAGGCGATAGAACCGACCAC; CG9596 Rev, GGCGGCCACCACCAGACTGT; RP49 Fwd, AGTGCGTCGCCGCTTCAAGG; and RP49 Rev, AGAACGCAGGCGACCGTTGG. RP49 refers to the gene ribosomal protein $\mathrm{L} 32$, which was used as an internal loading control in all experiments. Amplified PCR products were detected using SYBR Green 1 reagents from Roche. The relative level of transcript was calculated using the following formula: ratio $=E^{\Delta C p}$ (target) $/ E^{\Delta C P}$, where $E$ is the efficiency of the PCR, calculated from the slope of a standard curve using the equation $E=10^{[-1 / \text { slope }]}$ (Pfaffl, 2001) and $\Delta C p$ is the difference between the control and mutant $C p$ values.

Electrophysiology. Larvae were dissected (Jan and Jan, 1976) in a hemolymph-like solution (HL3.1) (Feng et al., 2004) containing $0.2 \mathrm{~mm}$ calcium and pinned to the Sylgard (Dow Corning) base of a $0.5 \mathrm{ml}$ perfusion bath. The segmental nerves exiting the ventral ganglion were cut and drawn into a fire-polished glass stimulating pipette. Square wave pulses $(0.15 \mathrm{~ms} ; 5-15 \mathrm{~V})$ were delivered to generate action potentials in the motor neurons. Electrophysiological recordings were obtained from muscles 6 and 7 of abdominal segments 3 and 4 with sharp intracellular microelectrodes filled with $1.5 \mathrm{M}$ potassium chloride and $1.5 \mathrm{M}$ potassium acetate (20-50 M $\Omega$ ). Stimulus evoked excitatory junction potentials (EJPs) and spontaneous miniature EJPs (mEJPs) were recorded using an Axoclamp 2B amplifier and 0.1 LU head stage from Molecular Devices. Analog signals were low-pass filtered at $5 \mathrm{kHz}$, digitized at $20 \mathrm{kHz}$ by a Powerlab/4SP A/D converter, and saved on a computer for later analysis using Mini Analysis software (Synaptosoft).

Pavlovian olfactory conditioning. Learning experiments were done as described by Tully and Quinn (1985). Three- to 5-d-old flies were sequentially exposed to two odors for $60 \mathrm{~s}$ each. The first odor was paired with electric shock $(60 \mathrm{~V}, 12 \times 1.5 \mathrm{~s}$ pulses $)$, whereas the second odor was not. Associative learning was tested within 3 min of completion of the training. During the testing, flies were exposed to both odors simultaneously in a T-maze. All training and testing were performed in a climate-controlled room with $75 \%$ humidity at $25^{\circ} \mathrm{C}$ under dim red light. The learning index was calculated as the percentage of flies that correctly avoided the odor paired with electric shock minus the percentage that incorrectly avoided the second odor. The paired and unpaired odors were swapped in each half of the experiment. The performance index (PI) was calculated as the average of the two reciprocal learning index values. Sensorimotor responses were tested in untrained flies of the same age. Flies were placed in a T-maze and given a choice between an odor and clean air or between two electric grids, with only one of the grids connected to the stimulator. Avoidance values represent the percentage of flies that avoided the odor or electric shock minus the percentage of flies that did not. The genotypes of experimental flies were coded before the experiments such that experiments were performed blind to avoid any subconscious bias.

Immunohistochemistry. Adult brains were dissected in phosphatebuffered solution with $0.1 \%$ Triton X-100 (PBST) and fixed in 4\% paraformaldehyde in PBST for $1 \mathrm{~h}$ at room temperature. Fixed brains were blocked overnight at $4^{\circ} \mathrm{C}$ with $4 \%$ normal goat serum and $4 \%$ normal donkey serum (filter sterilized) in PBST. Monoclonal primary antibodies against Fasciclin II (Fas II) and TRIO were obtained from the Developmental Studies Hybridoma Bank and used at a concentration of 1:10. The rabbit anti-green fluorescent protein (GFP) antibody was obtained from Invitrogen and used at 1:1000. Secondary antibodies (goat anti-rabbitAlexa Fluor 488 and donkey anti mouse-Cy3) were obtained from Invitrogen and used at a concentration of 1:1000. Stained brains were mounted on glass slides and imaged using a Zeiss Axiovert 200 equipped with a Hamamatsu Orca AG CCD camera and spinning disk confocal scan head driven by Volocity acquisition software.

Calcium imaging. The calcium indicator OGB (Oregon Green BAPTA1-dextran) was loaded into motor neuron boutons using the forward filling technique previously described (Macleod et al., 2002). Calcium levels in the boutons were measured by line scanning through individual type Ib boutons with a Bio-Rad 600 confocal scan head attached to a Nikon Optiphot- 2 microscope with an Olympus $40 \times$ water-immersion lens (0.7 numerical aperture). Line scans were captured at a rate of $4 \mathrm{~ms}$ per line. Images were analyzed using ImageJ software. The changes in fluorescence caused by calcium influx were plotted as $\Delta F / F_{\mathrm{o}}$, where $\Delta F$ is the change in fluorescence that occurs during axon stimulation and $F_{\mathrm{o}}$ is the resting fluorescence in the bouton before stimulation (calculated as the average fluorescence intensity within the region of interest minus the average fluorescence intensity in an adjacent background region).

Statistics. Two-tailed $t$ tests were used to compare two groups and ANOVAs followed by a Tukey post hoc test were used to compare multiple groups. All statistics were performed using Excel and SigmaPlot software. In all cases, the data are shown as \pm SEM.

\section{Results}

\section{Generation and characterization of ent 2 mutants}

The P124 insertion line (referred to from here on as ent $2^{\mathrm{P} 124}$ ) was originally generated in a screen looking for novel genes involved in learning and memory in Drosophila (Kamyshev et al., 2000; Bragina and Kamyshev, 2003). In these studies, the ent $2^{\mathrm{P} 124} \mathrm{mu}-$ tant was shown to have a defect in conditioned courtship suppression. Sequencing of the insertion site showed that the ent $2^{\mathrm{P} 124}$ insertion maps to position $26 \mathrm{E} 3$ on the left arm of the 
A

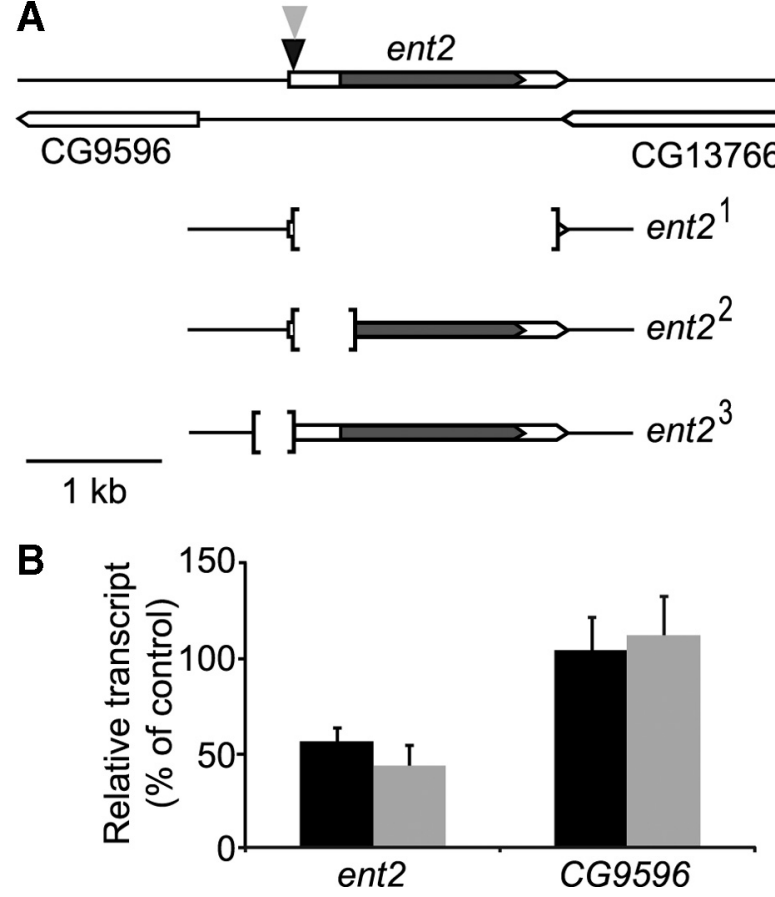

Figure 1. Characterization of ent2 mutant alleles. $\boldsymbol{A}$, The genomic region surrounding ent2. The coding region of ent2 is indicated by the shaded area. The ent ${ }^{\mathrm{P} 124}$ insertion is indicated by the black triangle, and the ent $2^{\text {Gal4 }}$ insertion is indicated by the gray triangle. The two nearest genes to ent2 (CG9596 and (G13766) are also depicted. Both (G9596 and (G13766 read 5' to 3' from right to left, whereas ent2 reads $55^{\prime}$ to $3^{\prime}$ from left to right. The imprecise excision mutants ent ${ }^{1}$, ent $2^{2}$, and ent $2^{3}$ are illustrated. The limits of the excisions are indicated by square brackets. $B$, Quantitative RT-PCR was performed on total RNA extracted from control, ent $2^{\mathrm{P} 124}$, and ent $2^{3}$ adult flies. The solid bars indicate the expression of ent2 and (G9596 in ent2 ${ }^{\mathrm{P} 124}$ flies normalized to control expression levels, whereas the shaded bars indicate the expression level of ent2 and (G9596 in ent $2^{3}$ mutants. Error bars indicate SEM.

second chromosome. As seen in Figure $1 A$, the ent $2^{\mathrm{P} 124}$ transposon is inserted in the $5^{\prime}$-untranslated region of ent2. The next closest gene to the insertion site is CG9596, $\sim 680$ bp away from the insertion site. To determine whether ent 2 was responsible for the phenotypes observed in the ent $2^{\mathrm{P} 124}$ insertion line, we performed an excision screen of the ent $2^{\mathrm{P} 124}$ transposon and generated two precise excisions and three independent imprecise excision mutants: ent $2^{1}$, ent $2^{2}$, and ent $2^{3}$ (Fig. $1 A$ ). The ent $2^{1}$ and $e n t 2^{2}$ excisions are both lethal during late larval/early pupal stages, whereas the ent $2^{3}$ excision is homozygous viable. Genetic complementation tests showed a failure to complement when the ent $2^{1}$ and ent $2^{2}$ mutant alleles were heterozygous to each other. Additionally, two independent deficiencies that covered the $26 \mathrm{E} 3$ region of the second chromosome [Df(2L)BSC6 and Df(2L)BSC7] both failed to complement the ent $2^{1}$ and ent $2^{2}$ mutants, indicating that the lethality in these mutants is attributable to the genetic excision of ent2, and not attributable to a second site mutation.

To determine whether the homozygous viable alleles (ent ${ }^{\mathrm{P} 124}$ and ent $2^{3}$ ) had any changes in ent 2 expression, we performed quantitative reverse transcription (qRT)-PCR on RNA extracted from control, ent $2^{\mathrm{P} 124}$, and ent $2^{3}$ adult flies. ent 2 expression was reduced in both ent $2^{\mathrm{P} 124}$ and ent $2^{3}$ flies relative to the $w^{1118}$ control (55.9 $\pm 10.3 \%$ of control in ent $2^{\mathrm{P} 124}, p<0.05 ; 43.4 \pm$ $7.4 \%$ of control in ent $2^{3}, p<0.05$ ) (Fig. 1 B). CG9596 expression, however, was normal in both ent $2^{\mathrm{P} 124}$ and ent $2^{3}$ mutants $\left(104.8 \pm 20.8 \%\right.$ of control in ent $2^{\mathrm{P} 124} ; 112 \pm 17.2 \%$ of control in ent $2^{3}$ ) (Fig. $1 B$ ). Both the ent $2^{1}$ and ent $2^{2}$ mutants showed un-
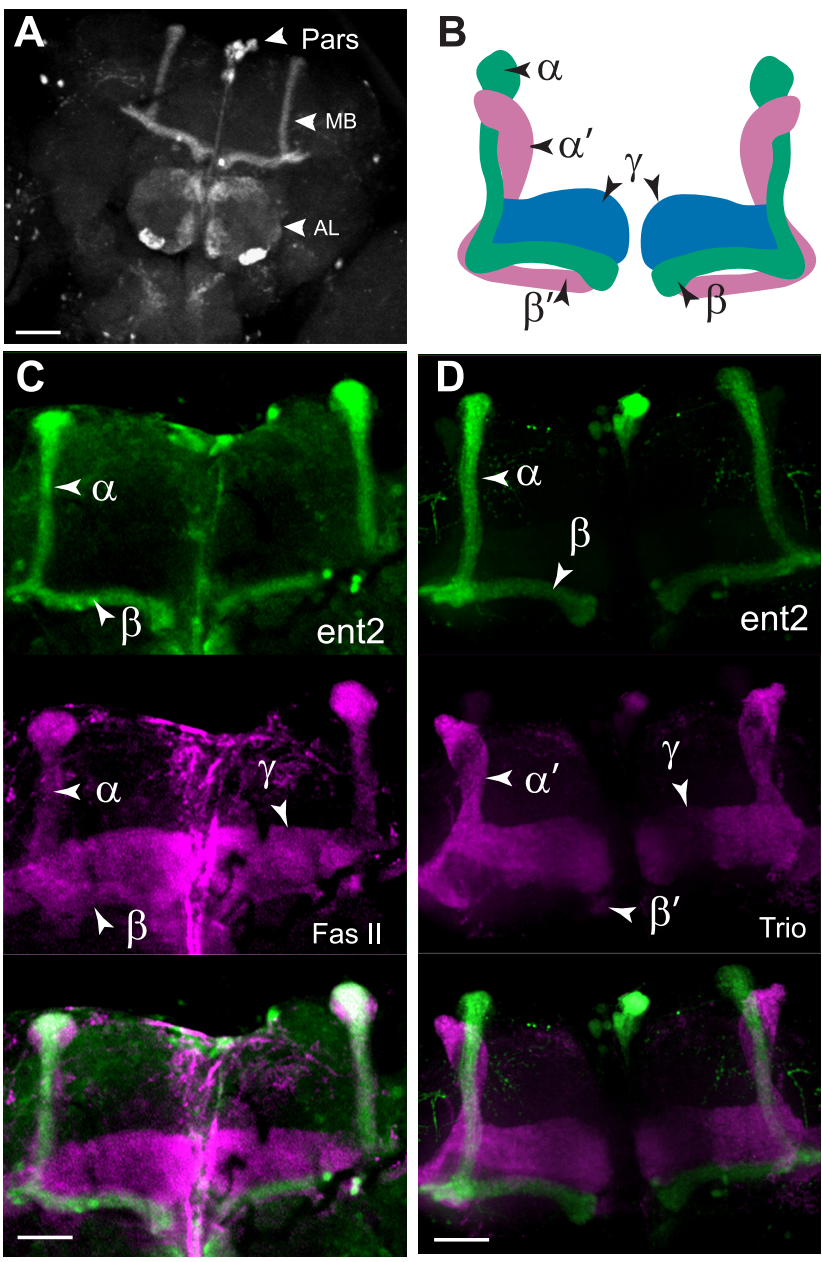

Figure 2. Expression of ent2. A, ent2 ${ }^{\text {Gal4 }}$ was used to drive expression of a UAS-eGFP transgene. In the adult CNS, eGFP expression is apparent in the pars, the MBs, and the ALs. $\boldsymbol{B}$, Model of the organization of the MB lobes. Axons travel from the Kenyon cells along the calyces (data not shown) where they split into three horizontal lobes $\left(\beta, \beta^{\prime}\right.$, and $\gamma$ ) and two vertical lobes $(\alpha$ and $\alpha^{\prime}$ ) that intertwine around each other. $C$, ent $2^{\text {Gal4 }}$-driven eGFP expression (top) colocalized with Fas II (middle) in the $\alpha / \beta$ lobes of the MB. D, ent $2^{\text {Gal4 }}$-driven eGFP expression (top) does not colocalize with Trio expression (middle) in the $\alpha^{\prime} / \beta^{\prime}$ or $\gamma$ lobes. Scale bars, $50 \mu \mathrm{m}$.

detectable levels of ent2 expression (data not shown). Together, these results show that ent $2^{1}$ and ent $2^{2}$ are genetic nulls, whereas ent $2^{\mathrm{P} 124}$ and ent $2^{3}$ are hypomorphic mutants. Neither the ent $2^{\mathrm{P} 124}$ insertion nor any of the excisions generated caused a significant change in the expression levels of CG9596, suggesting that the phenotypes observed in these mutants are specific and attributable to a reduction in ent 2 expression.

\section{ent 2 is expressed in the mushroom bodies and the} antennal lobes

Previous studies have shown both visual and cognitive defects in the ent $2^{\mathrm{P} 124}$ line (Kamyshev et al., 2000; Molotova-Besedina et al., 2009); however, the cellular and molecular mechanisms underlying these defects have not yet been characterized. We first examined the expression of ent2 in the CNS using the Gal4-UAS binary expression system (Brand and Perrimon, 1993) to drive expression of a UAS-enhanced GFP (eGFP) transgene under the control of the ent $2^{\text {Gal4 }}$ line. In the adult CNS, eGFP expression was observed primarily in the mushroom bodies (MBs), the antennal lobe (AL), and the pars intercerebralis (pars) (Fig. 2). We observed weak eGFP expression throughout the antennal lobes, 
with strong eGFP expression in a subset of glomeruli within the $\mathrm{AL}$ (Fig. $2 \mathrm{~A}$ ). Within the $\mathrm{AL}$, ent $2^{\mathrm{Gal} 4}$ driven eGFP expression appeared to be strongest in the VL1, VM4, VC3, and DM2 glomeruli (Laissue et al., 1999). The specific identity of the eGFPpositive glomeruli was confirmed by costaining antennal lobes with an antibody against bruchpilot (nc82) (Laissue et al., 1999) (data not shown). Studies of the anatomy of the Drosophila olfactory system have shown that most glomeruli are innervated by olfactory sensory neurons expressing a single olfactory receptor gene (Couto et al., 2005; Fishilevich and Vosshall, 2005). The DM2 glomerulus is innervated by olfactory receptor neurons expressing the Or22a olfactory receptor gene (Couto et al., 2005; Fishilevich and Vosshall, 2005). The identity of the olfactory receptor neurons innervating the VM4, VL1, and VC3 glomeruli remains to be determined, although it has been suggested that these glomeruli are innervated by olfactory receptor neurons originating in the coeloconic sensilla (Couto et al., 2005). The functional significance of the relatively restricted expression of ent 2 in the antennal lobes remains to be determined. In addition to the AL expression, we also observed strong expression within the MB. Figure 2, $C$ and $D$, shows colocalization of ent $2^{\text {Gal4 }}$ driven eGFP expression with Fasciclin II (Fas II) but not with TRIO. Antibodies against Fas II label the $\alpha / \beta$ and $\gamma$ lobes of the $\mathrm{MB}$, whereas antibodies against TRIO label the $\alpha^{\prime} / \beta^{\prime}$ and $\gamma$ lobes (Awasaki et al., 2000; Krashes et al., 2007). The results show that ent2 is expressed specifically in the $\alpha / \beta$ lobes of the mushroom bodies. Both the antennal lobes and the mushroom bodies are important structures for learning and memory in flies (Berry et al., 2008).

Fluorescent in situ hybridization experiments (FISH) showed that ent $2^{\text {Gal4 }}$-driven expression of eGFP in the larval CNS colocalized with the expression of a digoxigenin-labeled riboprobe specific to ent2 (supplemental Fig. 1, available at www.jneurosci. org as supplemental material). Additionally, we observed strong FISH labeling in frozen sections of the adult CNS in a pattern that reflects previous reports of dunce and rutabaga localization in the Kenyon cell bodies (supplemental Fig. 1, available at www. jneurosci.org as supplemental material) (Nighorn et al., 1991; Han et al., 1992). Together, these results suggest that the ent $2^{\text {Gal4 }}$ expression is a reliable indicator of endogenous ent 2 expression. Interestingly, when we used a two times higher concentration of ent2-specific riboprobe on frozen sections of the adult CNS, we were able to detect labeling in several cells in the optic lobe in addition to the labeling in the Kenyon cells (supplemental Fig. $1 F$, available at www.jneurosci.org as supplemental material). It is unclear whether this is indicative of a low-level expression of ent 2 expression in the optic lobes or simply nonspecific staining.

Of the four ENTs in mammals, ENT1, ENT2, and ENT4 are reported to show plasma membrane localization, whereas ENT3 shows an intracellular localization (Young et al., 2008). Given that both the $\mathrm{MB}$ and the $\mathrm{AL}$ where ent2 is most strongly expressed are dense neuropil structures, it would be extremely difficult to reliably determine whether ent 2 is expressed at the plasma membrane in these areas. To address this issue, we generated a V5-tagged ent 2 construct and expressed this construct in S2 cells under the control of the metallothionein promoter. As shown in supplemental Figure $1 H$ (available at www.jneurosci. org as supplemental material), the V5-tagged ent2 shows strong plasma membrane localization in transfected cells, but not in nontransfected cells (supplemental Fig. 1I, available at www. jneurosci.org as supplemental material). Previous work has shown ent2 is insensitive to pharmacological inhibitors (Machado et al., 2007). Combined with the plasma membrane localization we have observed for ent2, the localization results of the present study suggest that ent2 in Drosophila may be a functional homolog of mammalian ENT2.

\section{ent 2 mutants show defects in associative learning}

The expression of ent2 in antennal lobes and mushroom bodies supports a role for ent2 in learning and/or memory. Previous studies have shown that the ent $2^{\mathrm{P} 124}$ mutant allele causes defects in associative learning (Kamyshev et al., 2000; MolotovaBesedina et al., 2009). The previous studies, however, did not provide any evidence showing that the behavioral phenotypes are related to ent 2 expression or function. Our molecular analysis shows that ent $2^{\mathrm{P} 124}$ and $e n t 2^{3}$ mutant alleles both cause similar reductions in the levels of ent 2 transcript. To determine whether changes in ent 2 expression affect associative learning, we examined olfactory-based associative learning in both the ent $2^{\mathrm{P} 124}$ and ent $2^{3}$ hypomorphic mutant alleles immediately after training. Since defects in sensory input may contribute to any observed defects in associative learning, we first measured the olfactory acuity and shock sensitivity of the ent 2 mutants and found the sensory preferences to be unimpaired (supplemental Table 1, available at www.jneurosci.org as supplemental material). As previously shown, the ent $2^{\mathrm{P} 124}$ insertion line showed a significantly $(p<0.001)$ reduced performance index relative to the control line (compare performance indices \pm SEM of $85.8 \pm 2.6$ in control, $N=8$, with $66.3 \pm 3.7$ in ent $2^{\mathrm{P} 124}, N=8$ ) (Fig. $3 A$ ). Interestingly, ent $2^{3}$ mutants also showed a significant $(p<$ 0.001 ) reduction in the performance index relative to the control $(61.4 \pm 3.4 ; N=8)$ (Fig. $3 A$ ). In comparison, the ent2 precise excision lines showed normal performance indices compared with the $w^{1118}$ control (data not shown). These results combined with the qRT-PCR results (Fig. $1 B$ ) provide the first direct evidence of a link between ent2 expression and associative learning in Drosophila. We additionally examined associative learning in the independently generated homozygous ent $2^{\text {Gal4 }}$ line and observed a similar decrease in associative learning compared with the control $(56.4 \pm 7.4 ; N=4)$ (Fig. $3 A$ ). This result showing a comparable behavioral phenotype in an independently generated mutant allele provides additional support for a specific role for ent2 in associative learning in Drosophila.

We next asked where ent 2 expression is required for normal associative learning. For this purpose, we used RNA interference (RNAi) (Fire et al., 1998) to knock down expression of ent2 in specific regions of the CNS. A transgenic line containing a UASdriven inverted repeat (Dietzl et al., 2007) (obtained from the Vienna Drosophila RNAi Center) specific to ent2 (referred to here as ent $2^{\mathrm{RNAi}}$ ) was first crossed to the ent $2^{\mathrm{Gal} 4}$ line. To control for positional effects of either the Gal4 insertion or the UAS-RNAi insertion, both the ent $2^{\mathrm{Gal} 4}$ and the ent $2^{\mathrm{RNAi}}$ lines were crossed to $w^{1118}$ and the heterozygous offspring were used as controls. Flies bearing both the ent $2^{\mathrm{RNAi}}$ and the ent $2^{\text {Gal } 4}$ transgenes (Fig. $3 B$, solid bar) showed a significant $(p<0.05)$ reduction in performance indices compared with either the ent $2^{\mathrm{RNAi}} /+$ (Fig. $3 B$, shaded bar) or ent $2^{\text {Gal4 }} /+$ (Fig. $3 B$, open bar) control lines (compare performance indices of $60.4 \pm 3.9$ in ent $2^{\mathrm{Gal} 4}$; ent $2^{\mathrm{RNAi}}, N=$ 6 , with $73.3 \pm 3.8$ in ent $2^{\mathrm{Gal} 4} /+, N=6$, or $72.4 \pm 3.6$ in ent $2^{\mathrm{RNAi}} /+, N=6$ ). Since the sensory inputs were not impaired in any of the lines used (supplemental Table 1, available at www. jneurosci.org as supplemental material), the observed reductions in the performance indices suggest a specific defect in associative learning. These results provide additional support for a role for ent 2 in associative learning. Furthermore, these results provide additional evidence that the ent $2^{\text {Gal4 }}$ expression pattern in the 


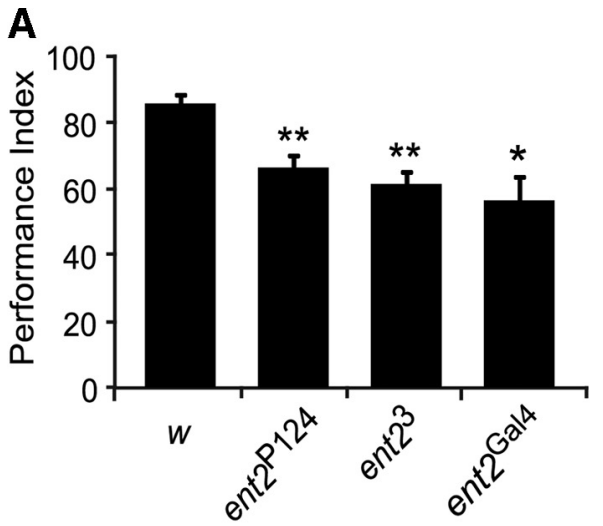

B
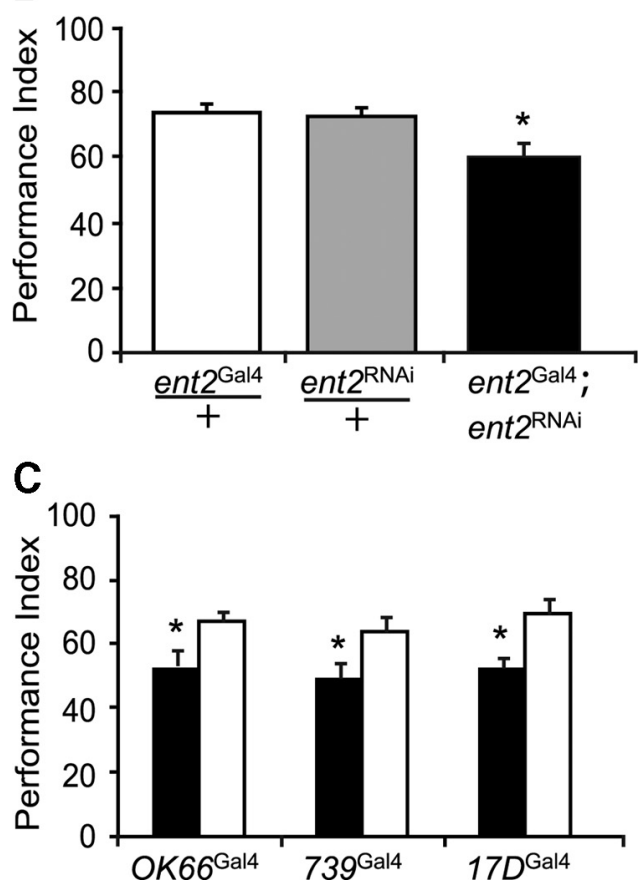

Figure 3. Associative learning defects in ent2 mutants. $A$, Both the ent $2^{\mathrm{P} 124}$ and the ent $2^{3}$ mutants showed a significant reduction in associative learning compared with the $w^{1118}(w)$ control $\left(N=8 ;{ }^{* *} p<0.001\right)$. We additionally observed a significant reduction in learning in homozygous ent $2^{\text {Gal4 }}$ flies $\left({ }^{*} p<0.05\right)$. $\boldsymbol{B}$, ent $2^{\text {Gal4 }}$ flies crossed to flies bearing an UAS-ent $2^{\text {RNAi }}$ construct showed reduced learning (solid bar) compared with flies bearing a single copy of either the ent ${ }^{\text {Gal4 }}$ enhancer alone (open bar) or the ent $2^{\text {RNAi }}$ transgene alone (shaded bar). $\boldsymbol{C}$, ent $2^{\text {RNAi }}$ flies were crossed to three different Gal4 enhancer trap lines, 0K66 ${ }^{\text {Gal4 }}$ (antennal lobe driver) and $739^{\text {Gal4 }}$ and $17 D^{\text {Gal4 }}$ (both mushroom body $\alpha / \beta$ lobe-specific drivers). Associative learning was impaired in the experimental lines (bearing both the ent2 ${ }^{\mathrm{RNAi}}$ transgene and the Gal4 enhancer) (solid bars) but not in flies bearing a single copy of the Gal4 enhancer alone (open bars). The asterisk $(*)$ indicates a significant difference, $p<0.05$. In all cases shown, the errors represent SEM.

adult CNS is a reliable indicator of endogenous ent 2 expression since knockdown of ent 2 expression specifically in these cells produced a similar phenotype to that observed in both the ent $2^{\mathrm{P} 124}$ and ent $2^{3}$ mutants.

Since ent 2 is expressed in both antennal lobes and mushroom bodies, and both of these structures have been implicated in learning/memory in Drosophila (Berry et al., 2008), we next asked which of these structures contributes to the observed defects. For this purpose, the ent $2^{\mathrm{RNAi}}$ line was crossed to different Gal4 enhancer trap lines to drive expression of the ent $2^{\mathrm{RNAi}}$ transgene specifically in the antennal lobes (OK66 ${ }^{\mathrm{Gal} 4}$ ) or in the mushroom bodies $\left(739^{\mathrm{Gal} 4}\right.$ or $\left.17 \mathrm{~d}^{\mathrm{Gal} 4}\right)$. Before the behavioral experiments, the expression patterns of these Gal4 enhancer traps were examined by crossing each line to the UAS-eGFP transgenic line. OK6 $6{ }^{\text {Gal4 }}$ drives eGFP expression primarily in the antennal lobes, although weak expression was observed in the core of the mushroom bodies and the pars intercerebralis (supplemental Fig. $2 A$, available at www.jneurosci.org as supplemental material), whereas both $739^{\mathrm{Gal} 4}$ and $17 \mathrm{D}^{\mathrm{Gal} 4}$ express strongly in the $\alpha / \beta$ lobes of the mushroom bodies (supplemental Fig. 2C,D, available at www.jneurosci.org as supplemental material). We observed a significant $(p<0.05)$ reduction in the performance indices when the ent $2^{\mathrm{RNAi}}$ line was crossed to OK66 ${ }^{\mathrm{Gal} 4}$ (compare performance indices \pm SEM of $53.1 \pm 5$ in OK6 $66^{\text {Gall }}$; ent $2^{\mathrm{RNAi}}, N=6$, with $67.3 \pm 2.9$ in $\left.\mathrm{OK} 66^{\mathrm{Gal} 4} /+, N=6\right)$. We also observed significant $(p<0.05)$ reductions in the performance indices when the ent $2^{\mathrm{RNAi}}$ line was crossed to either the $739^{\mathrm{Gal} 4}(49.3 \pm 4.5$ in $739^{\mathrm{Gal} 4}$; ent $2^{\mathrm{RNAi}}, N=6$, with $67.3 \pm 2.9$ in $739^{\mathrm{Gal} 4} /+, N=6$ ) or $17 \mathrm{D}^{\mathrm{Gal} 4}$ (compare $52.6 \pm 2.9$ in $17 \mathrm{D}^{\mathrm{Gal} 4}$; ent ${ }^{\mathrm{RNAi}}, N=6$, with $69.5 \pm 4.3$ in $17 \mathrm{D}^{\mathrm{Gal} 4} /+, N=6$ ) lines compared with their respective genetic controls (Fig. $3 C$ ). The above results suggest that a reduction in ent 2 expression in either the $\mathrm{AL}$ or the $\alpha / \beta$ lobes of the $\mathrm{MB}$ can lead to a reduction in associative learning. It should be noted, however, that whereas OK6 $6{ }^{\text {Gal4 }}$ drives expression primarily in the antennal lobes, we did observe some expression in the $\mathrm{MB}$ as well (supplemental Fig. $2 \mathrm{~A}$, available at www. jneurosci.org as supplemental material) such that we cannot rule out the possibility that the reduced performances in OK66 ${ }^{\mathrm{Gal} 4}$; ent $2^{\mathrm{RNAi}}$ flies may be the result of reduced ent 2 expression in the core of the MB.

Synaptic strength and plasticity are impaired in ent 2 mutants Previous studies on other learning and memory mutants have shown defects in synaptic transmission at the larval neuromuscular junction (Zhong and Wu, 1991; Zhong et al., 1992; Zhao et al., 2009). To determine whether ent 2 mutants had any defects in synaptic transmission, we measured synaptic strength and plasticity at the neuromuscular junction (NMJ) of third-instar larvae. We first examined spontaneously occurring transmitter release. The amplitude of mEJPs in either the ent $2^{\mathrm{P} 124}$ or ent $2^{3}$ mutants was not significantly different from the $w^{1118}$ control line $(0.84 \pm$ $0.03 \mathrm{mV}$ in control, $N=25 ; 0.86 \pm 0.04 \mathrm{mV}$ in ent ${ }^{\mathrm{P} 124}, N=21$; $0.92 \pm 0.03 \mathrm{mV}$ in ent $2^{3}, N=22$ ) (Fig. $4 A, B$ ). The frequency of mEJPs, however, was significantly $(p<0.001)$ elevated in both ent $2^{\mathrm{P} 124}$ and ent $2^{3}$ mutants relative to the control $(1.2 \pm 0.1 \mathrm{~Hz}$ in control, $2.3 \pm 0.2 \mathrm{~Hz}$ in ent $2^{\mathrm{P} 124}$, and $2.3 \pm 0.2 \mathrm{~Hz}$ in ent $\left.2^{3}\right)$. The amplitude of mEJPs may be affected by both presynaptic and postsynaptic factors (Karunanithi et al., 2002; Vautrin and Barker, 2003), whereas the frequency is affected exclusively by presynaptic factors. As such, the lack of change in the amplitude of mEJPs combined with an increase in the frequency of mEJPs is indicative of a presynaptic role for ent2 in synaptic transmission. We next looked at the amplitude of stimulus evoked transmitter release and observed a significant $(p<0.001)$ increase in the size of stimulus evoked EJPs in both ent $2^{\mathrm{P} 124}$ and ent $2^{3}$ mutants relative to the control (11.4 $\pm 0.9 \mathrm{mV}$ in control, $19.1 \pm 1.1 \mathrm{mV}$ in ent $2^{\mathrm{P} 124}$, and $18.4 \pm 1.1 \mathrm{mV}$ in ent $2^{3}$ ) (Fig. $4 C, D$ ). Given that we did not observe an increase in the amplitude of mEJPs, this increase in the amplitude of stimulus evoked EJPs indicates an increase in the number of vesicles released after arrival of the action potential at the NMJ.

We next looked at the effect of ent2 mutations on short-term synaptic plasticity. We first examined paired-pulse plasticity. Pairs of stimuli were delivered at different interstimulus intervals, and the ratio of the second (test) pulse to the first (conditioning) 
pulse was examined. Under the conditions used in the present study, we observed a marked facilitation of the test pulse with short interstimulus intervals in control preparations (Fig. 5A,B). Although the conditioning pulse was larger in ent $2^{\mathrm{P} 124}$ and ent $2^{3}$ mutants (Fig. 5A), the test pulse did not show the same degree of facilitation as seen in control preparations (Fig. 5B). Given that the first EJP in the ent 2 mutants is quite large relative to controls, we considered the possibility that the lack of paired-pulse facilitation in ent2 mutants was attributable to a saturation of postsynaptic receptors. To test this possibility, we examined a different form of short-term plasticity, specifically, posttetanic potentiation (PTP). Previous studies have shown that EJP amplitudes are transiently elevated after a brief tetanic stimulation (Zhong and $\mathrm{Wu}, 1991$; Knight et al., 2007). We compared the EJP amplitudes during a $0.2 \mathrm{~Hz}$ train of stimuli before and after a brief tetanic stimulation $(30 \mathrm{~s} ; 10 \mathrm{~Hz})$. EJP amplitudes were significantly higher in ent 2 mutants both before and after the tetanus relative to the controls such that the PTP ratio was not significantly different in the ent2 mutants relative to the control (compare PTP ratios \pm SEM of $1.6 \pm 0.23$ in $w^{1118}, N=16$, with $1.5 \pm 0.06$ in ent $2^{\text {P124 }}$, $N=16$, and $1.5 \pm 0.1$ in ent $2^{3}, N=17$ ) (Fig. $5 C$ ). These data demonstrate that ent 2 mutants do not have defects in PTP. Moreover, the increase in EJP amplitude after the tetanus cannot be attributed to changes in the density or function of postsynaptic receptors since the amplitude of mEJPs did not change after the tetanus (data not shown). The results of the PTP experiments show that both control and ent 2 mutants can facilitate the amplitude of the postsynaptic response independently of changes in postsynaptic receptor density. As such, the large EJPs and reduced paired-pulse facilitation observed in ent 2 mutants must be attributable to aberrant presynaptic transmitter release mechanisms.

\section{Calcium influx is elevated in ent 2 mutants}

The increased amplitude of stimulus evoked transmitter release combined with reduced paired-pulse facilitation in ent 2 mutants are consistent with an increase in transmitter release probability (Zucker and Regehr, 2002). Since the probability of transmitter release is strongly linked to changes in calcium influx (Fatt and Katz, 1952; Del Castillo and Katz, 1954; Neher and Sakaba, 2008), we asked whether stimulus-induced calcium influx is increased in ent 2 mutants. For this purpose, cut motor neurons were forward filled with the calcium-sensitive dye Oregon Green-1 BAPTA. Action potentials evoked in loaded axons led to a transient increase in fluorescence because of an increase in the cytosolic calcium concentration in synaptic boutons (Fig. 6A,B). We examined calcium responses during short trains of $2 \mathrm{~Hz}$ stimuli delivered in $2 \mathrm{~mm}$ extracellular calcium. Under these conditions, we observed significantly larger calcium transients in ent $2 \mathrm{mu}-$ tants relative to the controls [compare $\Delta F / F_{\mathrm{o}}$ values of $0.17 \pm$ 0.01 arbitrary units (au) in $w^{1118}, N=13$, with $0.22 \pm 0.01$ au in ent $2^{\text {P124 }}, N=10$, and $0.28 \pm 0.02$ au in ent $2^{3}, N=5$ ] (Fig. $6 C$ ). An increase in calcium response amplitude may reflect an in- crease in calcium influx or impairment in calcium clearance (Lnenicka et al., 2006; Klose et al., 2009). When we examined the decay constants of the calcium responses, however, we observed no significant differences between the ent 2 mutants and controls (compare decay constants of $17.5 \pm 2.4 \mathrm{~ms}$ in $w^{1118}$ with $18.6 \pm$ $1.6 \mathrm{~ms}$ in ent $2^{\mathrm{P} 124}$ and $17.1 \pm 2.9 \mathrm{~ms}$ in ent $2^{3}$ ) (Fig. 6D). These results show that extrusion of calcium from nerve terminals is not altered in ent 2 mutants, suggesting that the increased amplitude of the calcium responses is attributable to increased calcium influx.

\section{Interaction between ent 2 and adenosine receptor function}

Studies in mammals have suggested that equilibrative nucleoside transporters modify behavior by altering the concentration of extracellular adenosine available for the activation of adenosine receptors (Choi et al., 2004; Chen et al., 2007). We hypothesized that the extracellular availability of adenosine may be altered in ent2 mutants, leading to aberrant adenosine receptor activation. To address this possibility, we examined synaptic physiology and learning in ent2 mutants containing an adenosine receptor $(A d o R)$ mutation $\left(A d o R^{1}\right)$ generated by homologous recombination (Dolezal et al., 2005). The AdoR ${ }^{1}$ mutants express a truncated form of the receptor with 278 aa (instead of the usual 774). It is unclear at present what functional properties remain in the truncated $A d o R^{1}$ mutants; however, these mutants have been shown to rescue lethality in adenosine deaminase mutants by impairing adenosine receptor signaling (Dolezal et al., 2005), suggesting that the $A d o R^{1}$ mutants constitute at least a partial loss of function. We crossed flies bearing either the ent $2^{\mathrm{P} 124}$ or the ent $2^{3}$ mutations to flies bearing the $A d o R^{1}$ mutation to generate flies that were homozygous for both the ent 2 mutant allele and the $A d o R^{1}$ mutant allele. These double-mutant flies were completely viable.

We first examined associative learning in $A d o R^{1}$ mutants and ent2-AdoR ${ }^{1}$ double mutants. When we looked at associative learning in the $A d o R^{1}$ mutants, we noted that, similar to ent2 mutants, $A d o R^{1}$ mutants showed reduced performance indices 


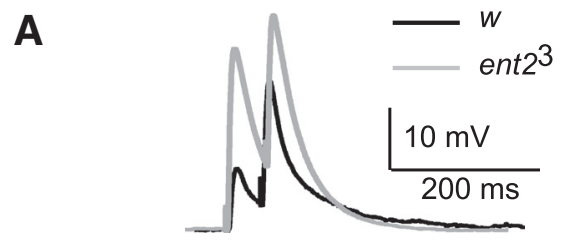

B
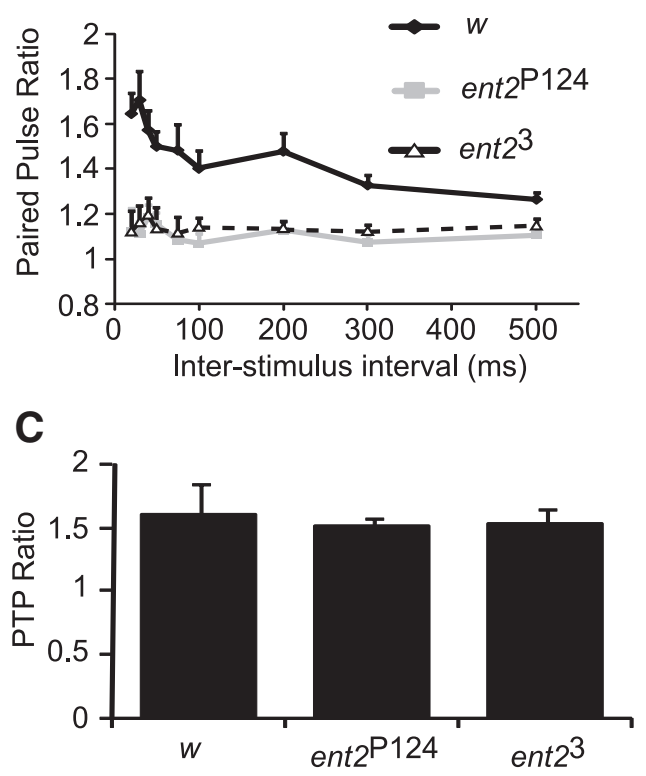

Figure 5. Synaptic plasticity is altered in ent2 mutants. $\boldsymbol{A}$, Example traces of paired-pulse experiments in $w^{1118}(w)$ control and ent $2^{3}$ mutants with a $50 \mathrm{~ms}$ interstimulus interval. $\boldsymbol{B}$, At short interstimulus intervals, $W^{1118}(w)$ control preparations (solid diamonds) showed marked paired-pulse facilitation (PPF), indicated by the higher paired-pulse ratios. This PPF declined as the interstimulus interval was increased. In ent $2^{\mathrm{P} 124}$ and ent $2^{3}$ mutants (shaded squares and open triangles, respectively), however, the paired-pulse ratio was significantly reduced, with the most dramatic differences observed at the shorter interstimulus intervals. C, The PTP ratio was calculated as the amplitude of posttetanic EJPs divided by the amplitude of pretetanic EJPs. Whereas the raw amplitude of both pretetanic and posttetanic EJPs was higher in ent ${ }^{3}$ and ent2 ${ }^{\mathrm{P} 124}$ lines relative to the control, the PTP ratio in these lines was not significantly different. Error bars indicate SEM.

compared with the control (compare $62.4 \pm 3.7$ in AdoR ${ }^{1}$ mutants, $N=7$, with $84.8 \pm 1.7$ in controls, $N=6$ ) (Fig. 7). Similarly, the performance indices in ent2-AdoR ${ }^{1}$ double mutants were also significantly reduced $(p<0.05)$ compared with the control but were not significantly different from performance indices of either the ent 2 or $A d o R^{1}$ mutants (performance indices: $54 \pm 5.3$ in ent $2^{\mathrm{P} 124} ; A d o R^{1}, N=8$, and $60.4 \pm 3.3$ in ent ${ }^{3}$; $\left.A d o R^{1}, N=8\right)$. These results suggest that the defects in associative learning observed in ent 2 mutants may be attributable to altered adenosine receptor activation.

We next examined synaptic physiology in the $A d o R^{1}$ mutants and the ent2-AdoR ${ }^{1}$ double mutants. Similar to our observations in the learning assay, $A d o R^{1}$ mutants showed defects in synaptic strength and plasticity similar to those observed in ent 2 mutants. First, EJP amplitudes were significantly elevated in $A d o R^{1} \mathrm{mu}-$ tants relative to the $w^{1118}$ control line (compare $19.6 \pm 3 \mathrm{mV}$ in Ado ${ }^{1}$ mutants, $N=10$, to $11.4 \pm 0.9 \mathrm{mV}$ in controls, $N=25$; $p<0.01$ ) (Fig. $8 A$ ). As with the ent2 mutants, the increased EJP amplitude in $A d o R^{1}$ mutants was attributable to a presynaptic change in transmitter release since the amplitude of the mEJPs in $A d o R^{1}$ mutants was not significantly different from the control (data not shown). Second, $A d o R^{1}$ mutants showed a reduction in paired-pulse plasticity at short interstimulus intervals similar to that seen in ent2 mutants [Fig. $8 \mathrm{~B}$, compare open magenta triangles $\left(A d o R^{1}\right)$ with solid yellow squares $\left(e n t 2^{\mathrm{P} 124}\right)$ and solid green triangles $\left.\left(e n t 2^{3}\right)\right]$. Finally, the amplitude of stimulus induced calcium transients was elevated in $A d o R^{1}$ mutants (compare $\Delta F / F_{\mathrm{o}}$ values of $0.17 \pm 0.01$ au in $w^{1118}, N=13$, with $0.24 \pm 0.02$ au in AdoR ${ }^{1}, N=13$ ) (Fig. 8C). As with ent 2 mutants, the increased amplitude of calcium transients in $A d o R^{1}$ was attributable to an increase in calcium influx rather than impaired calcium extrusion since the decay constants in $A d o R^{1}$ mutants were not significantly different from those in the controls (compare $17.5 \pm 2.3$ $\mathrm{ms}$ in control with $16.4 \pm 2 \mathrm{~ms}$ in $A d o R^{1}$ ). These results are consistent with our observations in the behavioral assay and suggest that the cognitive and synaptic phenotypes in ent 2 mutants may be attributable to decreased adenosine receptor activation. In contrast to our results with the behavioral assay, however, the synaptic defects observed in ent 2 and $A d o R^{1}$ mutants were rescued in ent2-AdoR ${ }^{1}$ double mutants. EJP amplitudes were not significantly different from the control in ent2-AdoR ${ }^{1}$ double mutants (compare $7.6 \pm 1.2 \mathrm{mV}$ in ent $2^{\mathrm{P} 124} ;$ AdoR $^{1}, N=16$, and $10.2 \pm 1.2 \mathrm{mV}$ in ent $2^{3} ;$ Ado $^{1}, N=15$, with $11.4 \pm 0.9 \mathrm{mV}$ in control) (Fig. $8 A$ ). ent2-Ado ${ }^{1}$ double mutants also showed normal paired-pulse facilitation [Fig. $8 B$, compare open blue squares $\left(\right.$ ent $\left.2^{\mathrm{P} 124} ; A d o R^{1}\right)$ and solid orange circles $\left(\right.$ ent $\left.2^{3} ; A d o R^{1}\right)$ with the solid black diamonds $\left.\left(w^{1118}\right)\right]$ and calcium influx (compare $\Delta F / F_{\text {o }}$ values of $0.16 \pm 0.01$ au in ent $2^{\mathrm{P} 124} ; A d o R^{1}$ with $0.17 \pm$ 0.01 au in control) (Fig. 8C).

We next asked whether compensatory changes in the expression of other nucleoside transporters might explain the similar synaptic phenotypes observed in ent 2 hypomorphic mutants and $A d o R^{1}$ truncated mutants despite normal synaptic strength and plasticity in the double mutants. To test this possibility, we examined the expression of all three equilibrative nucleoside transporters and both concentrative nucleoside transporters in ent2 and $A d o R^{1}$ mutants using quantitative RT-PCR. We observed a dramatic increase in the expression of ent 2 in $A d o R^{1}$ mutants (Fig. $8 D$ ) without any significant change in the expression of other nucleoside transporters in the $A d o R^{1}$ mutants (supplemental Fig. $4 A$, available at www.jneurosci.org as supplemental material). Furthermore, none of the other nucleoside transporters were transcriptionally upregulated in ent 2 mutants to compensate for the reduced ent 2 expression. In fact, we saw a significant decrease in the expression of ent1, ent 3 , and CNT1 in the ent2 mutants. Given that the $A d o R^{1}$ mutants display the same behavioral and synaptic phenotypes as ent2 mutants, yet do not show any changes in the expression of these genes, it seems unlikely that these changes in the expression of these nucleoside transporters can account for the phenotypes observed in the present study.

The dramatic increase in ent 2 expression observed in $A d o R^{1}$ mutants led us to ask whether ent2 expression is also increased in ent2-AdoR ${ }^{1}$ double mutants. Indeed, we found that the expression of ent 2 is also significantly increased in ent2-AdoR ${ }^{1}$ double mutants relative to the control (Fig. 8D). Although the expression of ent 2 in the double mutants was increased relative to the control, it was also significantly less than in $A d o R^{1}$ mutants alone. Together, these results suggest that the $A d o R^{1}$ truncation results in a large compensatory increase in ent 2 expression. When this effect is combined with the ent 2 hypomorphic mutations, the net effect is an approximately threefold increase in ent2 expression relative to the control, or a $\sim 70 \%$ reduction relative to the $A d o R^{1}$ mutant. These results suggest some form of cross talk exists between ent 2 expression and $A d o R$ function. The molecular consequences of the $A d o R^{1}$ truncation with respect to the coupling of 
the AdoR to intracellular signaling cascades are presently unclear. It is possible that the increase in ent 2 expression in these mutants may reflect a compensatory mechanism in response to a decrease or change in signaling through the AdoR. In the ent2-AdoR ${ }^{1}$ double mutants, this compensatory increase in ent 2 expression is tempered by the presence of the ent 2 hypomorphic mutant alleles.

In addition to the changes in expression of other nucleoside transporters, we also observed a significant reduction in the expression of several members of the adenosine deaminase-related growth factor (ADGF) family of genes in both ent2 and $A d o R^{1}$ mutants (supplemental Fig. $4 B$, available at www.jneurosci.org as supplemental material). Several of the ADGFs have been shown to possess strong adenosine deaminase activity in flies (Zurovec et al., 2002). Given that the primary function of adenosine deaminases is to metabolize extracellular adenosine, the decreased expression of ADGFs may be indicative of a decrease in the extracellular concentration of adenosine.

\section{Synaptic defects in ent 2 mutants occur independently of changes in cAMP}

The above results suggest that there is an interaction between ent 2 and AdoR function; however, the nature of that interaction is unclear. Both the ent 2 hypomorphic and $A d o R^{1}$ truncated mutants show similar defects in synaptic strength and plasticity, yet double mutants appear normal. Based on these observations, we considered the possibility that the observed defects in synaptic strength and plasticity may be attributable to changes in cAMP metabolism since activation of the Drosophila AdoR leads to an increase in cAMP concentration in CHO cells (Dolezelova et al., 2007). Several previous studies have shown that genetic and pharmacological treatments that increase cAMP concentration lead to an increase in transmitter release and decrease in synaptic plasticity (Zhong and Wu, 1991; Cheung et al., 1999, 2006; Kuromi and Kidokoro, 2000; Yoshihara et al., 2000). It is possible, therefore, that the increased transmitter release observed in ent 2 mutants is attributable to an increase in adenosine receptor activation leading to an increase in the cAMP concentration. To test this hypothesis, we performed an enzymatic immunoassay (EIA) to measure cAMP concentrations in control and ent 2 mutants (supplemental Fig. 3A, available at www.jneurosci.org as supplemental material). Using this technique, we were unable to detect any significant differences in the cAMP concentration in our ent 2 mutants, despite seeing a robust increase in the cAMP concentration in the cAMP-specific phosphodiesterase mutant dunce ( $d n c)$.

A major caveat with the cAMP EIA is that it measures the average CAMP concentration in a whole tissue (or whole animal). Given the relatively limited expression pattern of ent2 (as highlighted in supplemental Fig. 1, available at www.jneurosci.org as supplemental material), it is possible that changes in the cAMP concentration in a small subset of cells might be masked by the lack of any changes in the majority of the tissue present in the

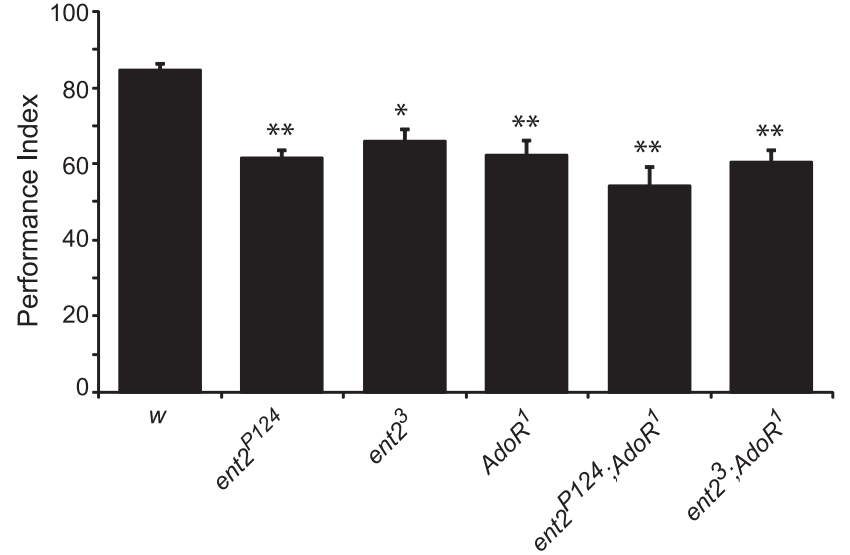

Figure 7. Associative learning is impaired in ent2 and $A d o R^{1}$ mutants. The performance indices in both $A d o R^{1}$ mutants and ent2-Ado $R^{1}$ double mutants were similar to those observed in ent 2 mutants. ${ }^{* *} p<0.01$ relative to the $w^{1118}$ control $(w)$; ${ }^{*} p<0.05$ relative to the control.

lysate. To address this possibility, we generated flies that were homozygous for the ent $2^{\mathrm{P} 124}$ mutation while carrying both an UAS-dunce transgene (UAS- $d n c$ ) and an inducible pan-neuronal driver (elav ${ }^{\text {Gal4-Pswitch }}$ ). Early third-instar larvae of this genotype were randomly separated into two treatment groups and allowed to develop to the wandering third-instar stage at $29^{\circ} \mathrm{C}$. The first group was placed in food containing the progesterone receptor antagonist RU486 (mifepristone) $(500 \mu \mathrm{M})$ to induce expression 
A

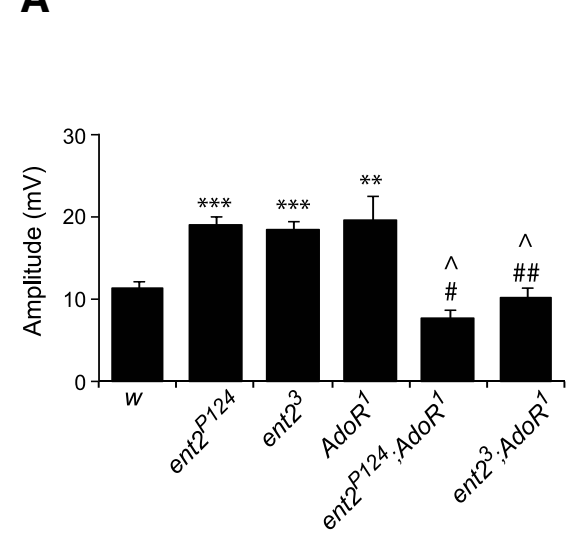

B

C

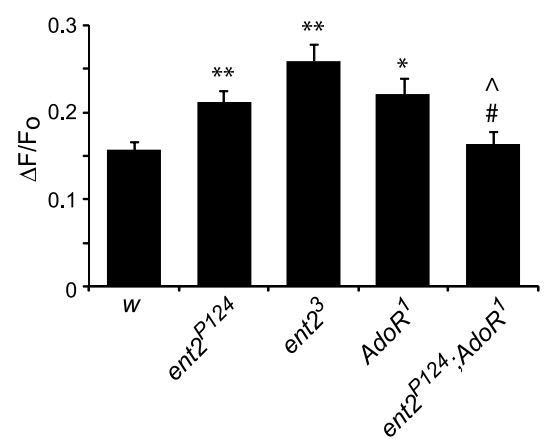

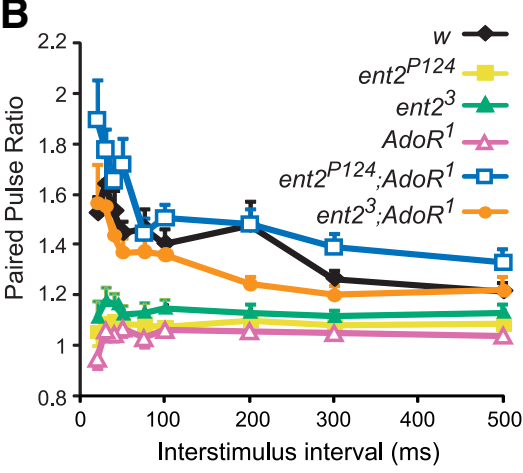

D

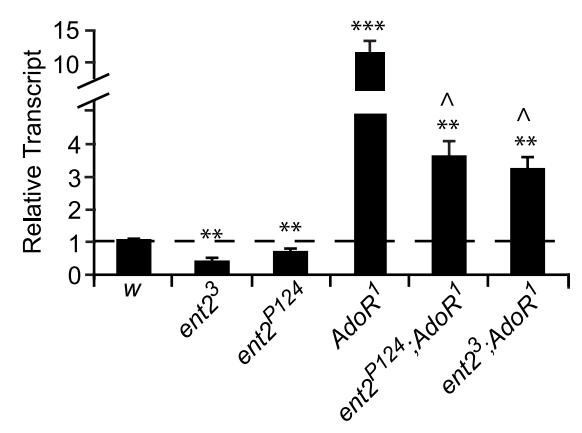

Figure 8. Synaptic defects in ent 2 and $A d o R^{1}$ mutants can be rescued by an $A d o R^{1}$ loss-of-function mutation. $A$, EJP amplitudes were elevated in ent2 and $A d o R^{1}$ mutants relative to the $w^{1118}$ control $(w)$ but not in ent2-AdoR ${ }^{1}$ double mutants. $B$, The paired-pulse ratio at short interstimulus intervals was reduced in both ent 2 and $A d o R^{1}$ mutants, but not in ent2-Ado ${ }^{1}$ double mutants. $C$, Both ent2 mutants and the $A d o R^{1}$ mutant showed significantly larger calcium transients relative to the $W^{1118}$ control $(w)$. The ent $2^{\mathrm{P} 124}$;Ado $R^{1}$ double mutant, however, showed normal calcium transients, not significantly different from the control. D, The expression of ent2 was examined by quantitative RT-PCR. As seen in Figure 1, ent2 expression is significantly reduced in ent2 mutants compared with the $w^{1118}$ control $(w)$. ent 2 expression was dramatically increased ( $>10$-fold) in Ado $R^{1}$ mutants and moderately increased ( $\sim 3$-fold) in ent2-Ado ${ }^{1}$ double mutants relative to the control. ${ }^{* *} p<0.001$ relative to control; ${ }^{* *} p<$ 0.01 relative to control; ${ }^{*} p<0.05$ relative to control; ${ }^{\#} p<0.05$ relative to ent $2^{\mathrm{P} 124} ;{ }^{\# \#} p<0.05$ relative to ent2 ${ }^{3} ; \wedge p<0.05$ relative to $A d o R^{1}$. Error bars indicate SEM.

of UAS- $d n c$, whereas the second group was placed in food containing a comparable volume of ethanol (vehicle). We then examined synaptic strength and plasticity at the NMJ in vehicle-fed (control) and mifepristone-fed larvae. If adenosine receptor activation is elevated in ent 2 mutants, leading to an increase in the concentration of cAMP, expression of a UAS- $d n c$ transgene in the ent $2^{\mathrm{P} 124}$ mutant background should rescue the increased synaptic strength. In contrast, however, the synaptic defects were amplified in mifepristone-fed larvae such that EJP amplitudes were elevated compared with vehicle-fed larvae (supplemental Fig. $3 B$, available at www.jneurosci.org as supplemental material). This increase in EJP amplitude was not accompanied by any significant changes in the amplitude of mEJPs (data not shown). We also noted a decrease in paired-pulse plasticity in mifepristonefed larvae compared with vehicle-fed larvae (supplemental Fig. $3 C$, available at www.jneurosci.org as supplemental material). These results, combined with the results of the cAMP EIA, are thus inconsistent with a model predicting increased cAMP concentrations in the ent 2 mutants generated by increased activation of the adenosine receptor.

\section{Discussion}

ENTs are evolutionarily conserved proteins that affect a diverse array of processes (Rose and Coe, 2008; Young et al., 2008). In mammals, ENT function regulates behaviors such as sleep/arousal states, drug dependence/addiction, and mood disorders (Alanko et al., 2003; Choi et al., 2004; Guillén-Gómez et al., 2004; Chen et al., 2007). ENTs are also therapeutic routes of entry for nucleoside analogues used in the treatment of diseases such as cancer and malaria (Zhang et al., 2007; Downie et al., 2008). As such, a detailed understanding of how ENT function affects these processes is of interest to a wide range of disciplines. In the present study, we have examined a novel role for ENTs in Drosophila. We showed virtually identical defects in associative learning in three independently generated mutant alleles of ent2. These reductions in associative learning were correlated with a decrease in the expression of ent 2 in both the ent $2^{\mathrm{P} 124}$ mutants and the newly generated ent $2^{3}$ mutants. Furthermore, knocking down ent2 expression using RNAi (Fire et al., 1998) also impaired associative learning. Together, these results provide compelling evidence to link ent 2 function to associative learning in Drosophila.

\section{Mutations in ent 2 elevate presynaptic calcium influx and transmitter release probability}

Several studies have demonstrated a correlation between synaptic defects observable at the larval neuromuscular junction and impaired learning in Drosophila (Zhong and Wu, 1991; Zhong et al., 1992; Zhao et al., 2009). In the present study, we observed an approximately twofold increase in the amplitude of stimulus evoked EJPs in ent $2^{\mathrm{P} 124}$ and ent $2^{3}$ mutants relative to the control. Several lines of evidence suggest that the observed differences in EJP amplitude originate in the presynaptic terminal. First, the amplitude of mEJPs was not significantly different in the ent 2 mutants, whereas the frequency of mEJPs was higher. Second, we also observed a reduction in paired-pulse plasticity, a phenomenon known to be attributable to changes in presynaptic calcium handling (Fisher et al., 1997; Zucker and Regehr, 2002; Neher and Sakaba, 2008). Finally, we observed an increase in stimulus-dependent calcium entry specifically in presynaptic boutons. Given the fourth-power relationship between calcium influx and transmitter release (Fatt and Katz, 1952; Del Castillo and Katz, 1954), the observed increase in stimulus-dependent calcium influx was entirely consistent with a twofold increase in transmitter release probability. Additionally, the correlation between the presynaptic calcium increases and the postsynaptic responses indicates that voltage-gated calcium channels at the active zone are the primary source of the increased calcium. Together, the results suggest that the increased probability of release and reduced paired-pulse plasticity observed in ent 2 mutants result from elevated calcium influx through voltage-gated calcium channels. 


\section{Interaction between ent 2 and adenosine receptor function}

In mammals, nucleoside transporters have been implicated in the regulation of several physiological processes via their ability to modulate the extracellular concentration of adenosine (Meester et al., 1998; Ackley et al., 2003; Choi et al., 2004; Chen et al., 2007). Our data suggest that the learning and synaptic defects observed in ent2 mutants may also be mediated by changes in adenosine receptor activation. We showed that ent 2 hypomorphic mutants and $A d o R^{1}$ truncated mutants have similar defects in both associative learning and synaptic function. The defects in synaptic function, however, were rescued in ent2-Ado ${ }^{1}$ double mutants. We also observed a dramatic increase in ent 2 expression in $A d o R^{1}$ mutants, suggesting the existence of a functional link between AdoR function and ent 2 expression. This cross talk between the receptor and the transporter appears to be specific to ent 2 since the expression of the other equilibrative or concentrative nucleoside transporters were not affected in $A d o R^{1}$ mutants.

Although the results of the present study indicate the presence of some form of cross talk between ent 2 and the $A d o R$, the nature of this cross talk is not clear. Studies in ENT1 knock-out mice suggest that loss of ENT1 leads to an increase in adenosine receptor activation in the amygdala and a decrease in adenosine receptor activation in the striatum (Choi et al., 2004; Chen et al., 2007). Measurements of extracellular adenosine concentration are technically challenging because of the unstable nature of adenosine. The task is even further complicated, however, by the possibility that extracellular adenosine levels may be variable even within different regions of the same organ. One way to circumnavigate this problem is to try to indirectly measure adenosine concentrations by measuring the level of adenosine receptor activation; however, this requires an understanding of the signaling pathways affected by adenosine receptor activation. In Drosophila, this task should be relatively simple because of the presence of a single adenosine receptor gene. When expressed in CHO cells, the AdoR produces an increase in cAMP and intracellular calcium in response to increasing concentrations of the agonist (Dolezelova et al., 2007). It is unclear at present whether similar signaling pathways are activated in vivo. We were unable to detect changes in cAMP in either ent 2 or $A d o R^{1}$ mutants, although the restricted expression pattern of ent 2 in the adult CNS may be masking changes in a small group of cells. Although the results of the present study did not support a role for cAMP in the observed defects in synaptic function, we cannot rule out a role for cAMP in the defects in associative learning. The fact that ent2-AdoR ${ }^{1}$ double mutants rescue the defects in synaptic function, but do not rescue the defects in associative learning, is suggestive of a different mechanism in the CNS that may still involve changes in cAMP. It should also be noted that ent 2 may also affect cAMP concentration independently of adenosine receptor activation by altering the intracellular concentration of adenosine available for synthesis of ATP and cAMP.

Although we were not able to directly or indirectly measure extracellular adenosine concentrations, the quantitative RT-PCR results may shed some light on the nature of the interaction between ent 2 and the AdoR. We noted a dramatic ( $>10$-fold) increase in the expression of ent2 in $A d o R^{1}$ mutants. This result suggests that ent 2 expression may be modulated by AdoR signaling. The nature of the signaling events linking AdoR activation and ent 2 expression as well as the interaction of the truncated $A d o R^{1}$ receptor with downstream signaling pathways remain to be determined. Regardless of the molecular link between $A d o R^{1}$ function and ent2 expression, however, these RT-PCR results suggest that ent 2 expression may be regulated to provide a con- stant adenosine tone. In the $A d o R^{1}$ mutants, the extracellular adenosine concentration is presumably normal, but as a result of the truncation, AdoR signaling is aberrant leading to a dramatic compensatory increase in the expression of ent2. If this model is correct, extracellular adenosine concentrations and thus adenosine tone may be reduced in ent 2 mutants. A similar reduction in adenosine tone was observed in the striatum of ENT1-null mice (Choi et al., 2004). Consistent with this hypothesis, expression of the adenosine deaminase-like growth factors, which have been shown to have strong adenosine deaminase activity (Zurovec et al., 2002), was reduced in ent 2 mutants. Additional work will be required to understand the molecular nature of the cross talk between ent 2 and AdoR in Drosophila; however, the results of the present study confirm that regulation of nucleoside transport is an evolutionarily conserved method for regulating purinergic signaling.

\section{References}

Ackley MA, Governo RJ, Cass CE, Young JD, Baldwin SA, King AE (2003) Control of glutamatergic neurotransmission in the rat spinal dorsal horn by the nucleoside transporter ENT1. J Physiol 548:507-517.

Alanko L, Stenberg D, Porkka-Heiskanen T (2003) Nitrobenzylthioinosine (NBMPR) binding and nucleoside transporter ENT1 mRNA expression after prolonged wakefulness and recovery sleep in the cortex and basal forebrain of rat. J Sleep Res 12:299-304.

Awasaki T, Saito M, Sone M, Suzuki E, Sakai R, Ito K, Hama C (2000) The Drosophila trio plays an essential role in patterning of axons by regulating their directional extension. Neuron 26:119-131.

Berry J, Krause WC, Davis RL (2008) Olfactory memory traces in Drosophila. Prog Brain Res 169:293-304.

Bragina YV, Kamyshev NG (2003) Comparative studies of four Drosophila P-insertion mutants with memory defects. Neurosci Behav Physiol 33:73-79.

Brand AH, Perrimon N (1993) Targeted gene expression as a means of altering cell fates and generating dominant phenotypes. Development 118:401-415.

Burnstock G (2008) Purinergic signalling and disorders of the central nervous system. Nat Rev Drug Discov 7:575-590.

Chen J, Rinaldo L, Lim SJ, Young H, Messing RO, Choi DS (2007) The type 1 equilibrative nucleoside transporter regulates anxiety-like behavior in mice. Genes Brain Behav 6:776-783.

Cheung U, Atwood HL, Zucker RS (2006) Presynaptic effectors contributing to cAMP-induced synaptic potentiation in Drosophila. J Neurobiol 66:273-280.

Cheung US, Shayan AJ, Boulianne GL, Atwood HL (1999) Drosophila larval neuromuscular junction's responses to reduction of cAMP in the nervous system. J Neurobiol 40:1-13.

Choi DS, Cascini MG, Mailliard W, Young H, Paredes P, McMahon T, Diamond I, Bonci A, Messing RO (2004) The type 1 equilibrative nucleoside transporter regulates ethanol intoxication and preference. Nat Neurosci 7:855-861.

Couto A, Alenius M, Dickson BJ (2005) Molecular, anatomical, and functional organization of the Drosophila olfactory system. Curr Biol 15:1535-1547.

Del Castillo J, Katz B (1954) Quantal components of the end-plate potential. J Physiol 124:560-573.

Dietzl G, Chen D, Schnorrer F, Su KC, Barinova Y, Fellner M, Gasser B, Kinsey K, Oppel S, Scheiblauer S, Couto A, Marra V, Keleman K, Dickson BJ (2007) A genome-wide transgenic RNAi library for conditional gene inactivation in Drosophila. Nature 448:151-156.

Dolezal T, Dolezelova E, Zurovec M, Bryant PJ (2005) A role for adenosine deaminase in Drosophila larval development. PLoS Biol 3:e201.

Dolezelova E, Nothacker HP, Civelli O, Bryant PJ, Zurovec M (2007) A Drosophila adenosine receptor activates cAMP and calcium signaling. Insect Biochem Mol Biol 37:318-329.

Downie MJ, Kirk K, Mamoun CB (2008) Purine salvage pathways in the intraerythrocytic malaria parasite Plasmodium falciparum. Eukaryot Cell 7:1231-1237.

Fatt P, Katz B (1952) Spontaneous subthreshold activity at motor nerve endings. J Physiol 117:109-128. 
Feng Y, Ueda A, Wu CF (2004) A modified minimal hemolymph-like solution, HL3.1, for physiological recordings at the neuromuscular junctions of normal and mutant Drosophila larvae. J Neurogenet 18:377-402.

Fire A, Xu S, Montgomery MK, Kostas SA, Driver SE, Mello CC (1998) Potent and specific genetic interference by double-stranded RNA in Caenorhabditis elegans. Nature 391:806-811.

Fisher SA, Fischer TM, Carew TJ (1997) Multiple overlapping processes underlying short-term synaptic enhancement. Trends Neurosci 20:170177.

Fishilevich E, Vosshall LB (2005) Genetic and functional subdivision of the Drosophila antennal lobe. Curr Biol 15:1548-1553.

Guillén-Gómez E, Calbet M, Casado J, de Lecea L, Soriano E, Pastor-Anglada M, Burgaya F (2004) Distribution of CNT2 and ENT1 transcripts in rat brain: selective decrease of CNT2 mRNA in the cerebral cortex of sleepdeprived rats. J Neurochem 90:883-893.

Han PL, Levin LR, Reed RR, Davis RL (1992) Preferential expression of the Drosophila rutabaga gene in mushroom bodies, neural centers for learning in insects. Neuron 9:619-627.

Jan LY, Jan YN (1976) L-Glutamate as an excitatory transmitter at the Drosophila larval neuromuscular junction. J Physiol 262:215-236.

Kamyshev NG, Iliadi KG, Bragina YV, Savvateeva-Popova EV, Tokmacheva EV, Preat T (2000) Identification of Drosophila mutant with memory defects after acquisition of conditioned reflex suppression of courtship. Neurosci Behav Physiol 30:307-313.

Karunanithi S, Marin L, Wong K, Atwood HL (2002) Quantal size and variation determined by vesicle size in normal and mutant Drosophila glutamatergic synapses. J Neurosci 22:10267-10276.

Klose MK, Boulianne GL, Robertson RM, Atwood HL (2009) Role of ATPdependent calcium regulation in modulation of Drosophila synaptic thermotolerance. J Neurophysiol 102:901-913.

Knight D, Iliadi K, Charlton MP, Atwood HL, Boulianne GL (2007) Presynaptic plasticity and associative learning are impaired in a Drosophila presenilin null mutant. Dev Neurobiol 67:1598-1613.

Krashes MJ, Keene AC, Leung B, Armstrong JD, Waddell S (2007) Sequential use of mushroom body neuron subsets during Drosophila odor memory processing. Neuron 53:103-115.

Kuromi H, Kidokoro Y (2000) Tetanic stimulation recruits vesicles from reserve pool via a cAMP-mediated process in Drosophila synapses. Neuron 27:133-143.

Laissue PP, Reiter C, Hiesinger PR, Halter S, Fischbach KF, Stocker RF (1999) Three-dimensional reconstruction of the antennal lobe in Drosophila melanogaster. J Comp Neurol 405:543-552.

Lima SQ, Miesenböck G (2005) Remote control of behavior through genetically targeted photostimulation of neurons. Cell 121:141-152.

Littleton JT, Ganetzky B (2000) Ion channels and synaptic organization: analysis of the Drosophila genome. Neuron 26:35-43.

Lnenicka GA, Grizzaffi J, Lee B, Rumpal N (2006) Ca2+ dynamics along identified synaptic terminals in Drosophila larvae. J Neurosci 26:1228312293.

Machado J, Abdulla P, Hanna WJ, Hilliker AJ, Coe IR (2007) Genomic analysis of nucleoside transporters in Diptera and functional characterization of DmENT2, a Drosophila equilibrative nucleoside transporter. Physiol Genomics 28:337-347.

Macleod GT, Hegström-Wojtowicz M, Charlton MP, Atwood HL (2002)
Fast calcium signals in Drosophila motor neuron terminals. J Neurophysiol 88:2659-2663.

Meester BJ, Shankley NP, Welsh NJ, Meijler FL, Black JW (1998) Pharmacological analysis of the activity of the adenosine uptake inhibitor, dipyridamole, on the sinoatrial and atrioventricular nodes of the guinea pig. Br J Pharmacol 124:729-741.

Molotova-Besedina NG, Iliadi NN, Bragina IuV, Kamysheva EA, Soboleva SA, Iliadi KG, Kamyshev NG (2009) Novel memory mutants in Drosophila: behavioral characteristics of the P-insertional mutant in the Ent2P locus (in Russian). Genetika 45:50-58.

Neher E, Sakaba T (2008) Multiple roles of calcium ions in the regulation of neurotransmitter release. Neuron 59:861-872.

Nighorn A, Healy MJ, Davis RL (1991) The cyclic AMP phosphodiesterase encoded by the Drosophila dunce gene is concentrated in the mushroom body neuropil. Neuron 6:455-467.

Pastor-Anglada M, Cano-Soldado P, Errasti-Murugarren E, Casado FJ (2008) SLC28 genes and concentrative nucleoside transporter (CNT) proteins. Xenobiotica 38:972-994.

Pfaffl MW (2001) A new mathematical model for relative quantification in real-time RT-PCR. Nucleic Acids Res 29:e45.

Rose JB, Coe IR (2008) Physiology of nucleoside transporters: back to the future. Physiology (Bethesda) 23:41-48.

Sankar N, Machado J, Abdulla P, Hilliker AJ, Coe IR (2002) Comparative genomic analysis of equilibrative nucleoside transporters suggests conserved protein structure despite limited sequence identity. Nucleic Acids Res 30:4339-4350.

Tully T, Quinn WG (1985) Classical conditioning and retention in normal and mutant Drosophila melanogaster. J Comp Physiol A Neuroethol Sens Neural Behav Physiol 157:263-277.

Vautrin J, Barker JL (2003) Presynaptic quantal plasticity: Katz's original hypothesis revisited. Synapse 47:184-199.

Yoshihara M, Suzuki K, Kidokoro Y (2000) Two independent pathways mediated by cAMP and protein kinase A enhance spontaneous transmitter release at Drosophila neuromuscular junctions. J Neurosci 20:8315-8322.

Young JD, Yao SY, Sun L, Cass CE, Baldwin SA (2008) Human equilibrative nucleoside transporter (ENT) family of nucleoside and nucleobase transporter proteins. Xenobiotica 38:995-1021.

Zhang J, Visser F, King KM, Baldwin SA, Young JD, Cass CE (2007) The role of nucleoside transporters in cancer chemotherapy with nucleoside drugs. Cancer Metastasis Rev 26:85-110.

Zhao H, Zheng X, Yuan X, Wang L, Wang X, Zhong Y, Xie Z, Tully T (2009) ben functions with scamp during synaptic transmission and long-term memory formation in Drosophila. J Neurosci 29:414-424.

Zhong Y, Wu CF (1991) Altered synaptic plasticity in Drosophila memory mutants with a defective cyclic AMP cascade. Science 251:198-201.

Zhong Y, Budnik V, Wu CF (1992) Synaptic plasticity in Drosophila memory and hyperexcitable mutants: role of cAMP cascade. J Neurosci 12:644-651.

Zucker RS, Regehr WG (2002) Short-term synaptic plasticity. Annu Rev Physiol 64:355-405.

Zurovec M, Dolezal T, Gazi M, Pavlova E, Bryant PJ (2002) Adenosine deaminase-related growth factors stimulate cell proliferation in Drosophila by depleting extracellular adenosine. Proc Natl Acad Sci U S A 99: 4403-4408. 